\title{
Mediating role of organization self-esteem in the relationship between perfectionism and mental health among Rafsanjan Oil Company staff (2017)
}

\author{
Ziaaddini M, $\mathrm{PhD}^{1^{*}}$, Alinezhad $\mathrm{H}, \mathrm{PhD}$ Student $^{2}$ \\ 1- Assistant Prof., Dept of Management, Rafsanjan Branch, Islamic Azad University,, Rafsanjan, Iran. 2- PhD Student, Dept \\ of Management, Rafsanjan Branch, Islamic Azad University, Rafsanjan, Iran.
}

\begin{abstract}
Received: March 2017, Accepted: October 2017

Background: The aim of this study was to examine the relationship between perfectionism and mental health according to the mediating role of organization self-esteem (OSE) in the official staff of the Iranian Oil Pipeline of the central region and Telecommunication Company of the southeast region, Rafsanjan, Iran.

Materials and Methods: This descriptive research is a path way analysis. The statistical population included all employees at the Iranian Oil Pipeline and Telecommunication Company. Based on the Guernsey and Morgan tables, 95 subjects were selected as the samples through random method. In order to collect data, we employed three sets of questionnaires (perfectionism, mental health, and OSE) that have a proper validity and reliability. The data were analyzed using SPSS and PLS.

Results: The significant coefficient of the route between OSE and mental health was 0.264 that was estimated less than 1.96. Thus, it can be reported that ther was not a significant association between OSE and mental health which means OSE did not affect mental health. Therefore, it can be said that perfectionism did not affect mental health with OSE as the mediator variable.

Conclusions: According to the importance of using the skill of perfectionism, it can be used in increasing mental health in the organization.
\end{abstract}

Keywords: Perfectionism, Mental Health, Self Esteem

\section{Introduction}

Identification of psychological variables, especially personality variables related to health and mental illnesses, has been of interest to the researchers in psychological areas. Some personality traits can play an important role in the etiology and the progression of disorders. These traits can indirectly make the individuals sick by creating unhealthy behaviors like smoking, drug abuse and insomnia (1). Perfectionism, as a personality trait, is one of the factors affecting mental health, and various studies have confirmed this relationship (2).

Perfectionism is a personality trait characterized by a set of high standards of performance, and is defined as negative selfevaluation, criticism, and self-blame (2).
Perfectionism is divided into two types of healthy and normal, and unhealthy and abnormal. In normal perfectionism, the perfectionist people enjoy doing hard and onerous work and when they feel that they are free in doing the activities, they do their best. By success in doing the tasks, they in turn, feel satisfaction (3). Studies have shown that perfectionism is a constant source of stress, which often leaves the individual with a feeling of failure. Perfectionists expect themselves to be flawless. These constant expectations are considered as a source of stress (4).

\footnotetext{
* Corresponding author: Mohammad Ziaaddini, Dept of Management, Rafsanjan Branch, Islamic Azad University, Rafsanjan, Iran

E-mail: m.ziaaddini@yahoo.com
} 
One of the structures that recently has interested the industrial and organizational psychologists is organizational self-esteem (OSE). OSE can be defined as the feelings of worth or value that employees feel within their workplace (5). OSE is a reflection of evaluation of adequacy and person's worthiness as a member of the organization (6). According to the Ante's idea (2009), staff with superior self-esteem level have more desire to positive attitudes in their working environment, better behavior with their colleagues with lower self-esteem level, and also have a superior organizational commitment (7).

Obviously, the mental disease is not specified to a stratum or a specific region and includes all of society's levels. In fact, nobody is secure from mental and social pressure (8). Thus, mental health can be known as a universal condition in which is in or out of working environments (9).

Therefore, considering the theoretical background of this study and lack of researches done in this field, this research attempts to examine the relationship between perfectionism with mental health regarding the mediator variable of OSE in the official staff of the Iranian Oil Pipeline Company and Telecommunication Company in Rafsanjan, Iran.

\section{Material and Methods}

This research is descriptive with path analysis way .This study used primary data collected through questionnaires. First, respondents were assured that all their responses in this study will be kept confidential. The study emphasized on close-ended questionnaires which were answered based on the Likert scale (4-point scales) to determine the level of interest and perception of each element available. All questionnaire items were analyzed using the SPSS software (version 21, IBM Corporation, Armonk, NY, USA) and structural equation modeling (SEM) using partial least square (PLS) software, version 3, in order to obtain descriptive statistics data. PLS is formed of two parts of the measurement sample and structural sample (10). In the first part, the measurement sample, which is internal reliability and validity of research variables and instruments, was studied. Composite reliability (CR) is a better scale to model structural equation than Cronbach's alpha because in calculating Cronbach's alpha of every variable all parameters are imputed in calculation by the same importance. While in calculating the $\mathrm{CR}$, a parameter with more factorial load is more important. This subject makes CR rates of a variable more reliable and exact than Cronbach's alpha. CR rate for every variable which was more than 0.7 and less than 0.6 showed the lack of reliability. Next scale to study fitness of measurement model was convergent validity which studies correlation rate of every variable with its questions (parameters). In this area, average variance extracted (AVE) scale was used. AVE rate for every variable is more than 0.4 (11). In the second part, structural sample and research hypotheses were used by studying $\beta$ coefficient and bootstrap method to calculate $\mathrm{Z}$ statistics rate in order to determine route coefficients significance. Route coefficients were used to determine the share of every independent variable to explain dependent variables variance.

The statistical population included all employees (130 staff) of Iranian Oil Pipeline Company and Telecommunication Company, in the southeast of Rafsanjan in 2016-2017. Based on the Guernsey and Morgan tables, 95 subjects were selected as the samples through random method.

To measure research construction, three questionnaires of perfectionism, mental health, and OSE were used. The questionnaire has been adapted from previous studies and modified to fit the current context. The questionnaires consisted of items related to distributed perfectionism (29 questions ), items related to distributed mental health (19 questions ), and items to measure OSE (18 
questions ). All the questionnaires had a proper validity and reliability. The questionnaire was scored on a 4-point modified Likert scale of strongly agree, agree, strongly disagree, and disagree with weights of $4,3,2$ and 1 , respectively.

\section{Results}

Measurement sample test, parameters with a factorial load less than 0.4 such as parameters 60, 66 and 69 of OSE variable, parameters 38,
46, 48 and 45 of mental health variable, parameters $1,2,4,5,6,11,12,15,21$ and 26 of perfectionism variable were omitted to analyze because there was no significant load for other variables.

CR and convergent validity: In table $1, C R$ and convergent validity of every variable are presented. Regarding bases mentioned in the text, rates of this table show enough credit of variables.

Table 1: Composite reliability (CR) and convergent validity of the variables

\begin{tabular}{|c|c|c|c|}
\hline Variables & OSE & Mental health & Perfectionism \\
\hline CR & 0.950 & 0.900 & 0.903 \\
\hline Convergent validity & 0.546 & 0.403 & 0.407 \\
\hline
\end{tabular}

CR: Composite reliability

OSE: Organizational self-esteem

Another important measure determined by divergent validity is the relationship between variables with their parameters comparing with their relationship with other variables, as the accepted divergent validity of a model explained that a variable in the model had more interaction with its parameters than other variables. To study divergent validity by comparing correlation rate of a structure with its parameters against correlation of that variable with other variables, method was used in which AVE square of one variable should be more than the correlation of that variable with other variables (11). As determined in table 2, AVE square rate of variables in present blocks was located in the main diameter that is more than correlation rate among them which are located in below blocks in left of the main diameter. However, it can be explained that in current research, variables in the model have more interaction with their parameters than another variable. In other words, model discriminant validity is suitable. Also, regarding correlation matrix, among variables, the highest correlation rate was related to the relationship between perfectionism and mental health.

Table 2: Correlation matrix and studying discriminant validity of research variables

\begin{tabular}{|c|c|c|c|}
\hline Variables & Mental health & OSE & Perfectionism \\
\hline Mental health & 0.635 & - & - \\
\hline OSE & -0.13 & 0.739 & - \\
\hline Perfectionism & -0.546 & 0.202 & 0.638 \\
\hline
\end{tabular}

OSE: Organization self-esteem

Structural sample and research hypotheses were used by studying $\beta$ coefficient and bootstrap method to calculate $\mathrm{Z}$ statistics rate in order to determine route coefficients significance. Route coefficients are used to determine the share of every independent variable to explain dependent variables variance (Table 3 ).

The rate of $\mathrm{R}^{2}$ explains the variance of the dependent variable by independent variables. It indicates the effect of an exogenous variable on an endogenous one. Three rates of 0.19 , 
0.33 and 0.67 are regarded as the base rate for weak, medium and strong $\mathrm{R}^{2}$. According to table $4, R^{2}$ rate was calculated as 0.04 and 0.30 for OSE and mental health respectively. Therefore, the rates were in relatively weak level for OSE and relatively medium level for mental health.

Perfectionism effects on mental health: As shown in table 3 , the significant coefficient of the route between perfectionism and mental health was 8.35 (according to 5\% error principle in rejecting hypothesis 0 for rates of 1.96 and -1.96 per model parameters) that is estimated more than 1.96. So, it can be explained that researcher's objective was approved by $95 \%$ confidence. Regarding negativity of route coefficient $(-0.535)$, it can be claimed that perfectionism has affected mental health negatively and significantly.

Table 3: Route coefficients, $\mathrm{Z}$ statistics

\begin{tabular}{|c|c|c|c|}
\hline Route & Z statistics & Effect coefficient & Significance level \\
\hline Perfectionism $\rightarrow$ mental health & 8.35 & -0.535 & $\mathrm{P}<0.05$ \\
\hline Perfectionism $\rightarrow$ OSE & 2.13 & 0.234 & $\mathrm{P}<0.05$ \\
\hline OSE $\rightarrow$ mental & 0.26 & -0.027 & $\mathrm{P}<0.05$ \\
\hline
\end{tabular}

OSE: Organizational self-esteem

\section{Perfectionism effects on mental health with} OSE as the mediator variable: As shown in table 3 , the significant coefficient of the route between perfectionism and OSE was 2.13 (according to 5\% error principle in rejecting (hypothesis 0) for rates of 1.96 and -1.96 per model parameters) that is estimated more than 1.96. So, it can be explained that researcher's objective was approved by $95 \%$ confidence. Regarding positivity of route coefficient (0.234), it can be said that perfectionism affects OSE positively and significantly. Also, the significant coefficient of the route between OSE and mental health was 0.264 (according to $5 \%$ error principle in rejecting hypothesis 0 for rates of 1.96 and -1.96 per model parameters) that is estimated less than 1.96. So, it can be explained that researcher's objective was not approved by $95 \%$ confidence. The OSE did not affect mental health. Thus, perfectionism did not affect mental health with OSE as the mediator variable.

Table 4: $\mathrm{R}^{2}$ rate for organizational self-esteem (OSE) and mental health

\begin{tabular}{|c|c|c|}
\hline Variable & Mental health & OSE \\
\hline $\mathbf{R}^{2}$ & 0.30 & 0.04 \\
\hline
\end{tabular}

OSE: Organizational self-esteem

\section{Discussion}

The results have shown that perfectionism has a negative impact on mental health. It was almost consistent with the study by Capan (12), Stoeber and Rambow (13), Steel (14) and Abbaspour et al (15). The results have shown that perfectionism has a direct impact on Organization based -Self esteem, It was almost consistent with findings reported by Pierce et al (16). They reported that decrease perfectionism raises the self-esteem of governmental employees. The results have shown that Organization based -Self esteem no impact on mental health. It was not almost consistent with the study by $\operatorname{Shek}(17)$,Ni et al(18), Kunikata(19), Fialko et al(20).

The potential reason for lack of association between OSE and mental health is that other studies have not studied some personal variables such as professional maturity, through which, the OSE may affect the mental health. 
and also results show that perfectionism has a negative impact on mental health, and decreases mental health in organizations. In this regard, no study was found to compare with the findings. Results of this research showed that self-esteem affects mental health(16)

Overall considering contradiction in different studies two conclusions can be achieved. First, other factors should be found in relationship between Organization based -Self esteem and mental health. Second, the sample size of the current work was small and accordingly, the modeled variables were not significant. Thus, some of these factors may be effective and it is suggested that larger sample size is considered in future work.

\section{Conclusion}

Based on the finding, perfectionism reduce organization based self-esteem and mental health. Thus, paying attention to the perfectionism and reduces it through various techniques such as continuous and related training courses workshops are of paramount importance.

\section{Acknowledgments}

Hereby, we express our gratitude to the managing director, research manager and staff for their support in performing this study.

Conflict of interest: None declared.

\section{References}

1. Mayer BW, Fraccastoro KA, Mcnary LD. The relationship among organizational based selfesteem and various factors motivating volunteers. Nonprofit and Voluntary Sector 2007; 36(2):32740.

2. Lee MS, Lee MB, Liao SC, Chiang FT. Relationship between mental health and job satisfaction among employees in a medical center department of laboratory medicine. J Formos Med Assoc 2009; 108(2):146-54.

3. Yousefi H. The relationship between organizational justice and organizational self esteem and aggression in Zarand city employees in executive [MSc thesis]. Rafsanjan: Azad University of Rafsanjan; 2012. P.41

4. Beheshtifar M. The relationship between procrastination and self-organizational behavior faculty members in seven regional units [MSc thesis]. Rafsanjan: Azad University in Rafsanjan; 2012. P.67

5. Adger WN, Arnell NW, Tompkins EL. Successful adaptation to climate change across scales. Glob Environ Change 2005; 15(2):77-86.

6. Ghafar Samar R, Shirazizadeh $M$. On the relationship between perfectionism, reading anxiety and reading Achievement: A study on the psychology of language learning. Language Related Research 2011; 2(1):1-19.

7. Nasri F. The relationship between mental capital and mental health nursing Kerman University of Medical Sciences [MSc thesis]. Rafsanjan: Azad University of Rafsanjan; 2012. P.72

8. Alinejad $\mathrm{h}$. Investigate the relationship between the employees and their mental health [MSc thesis]. Azad University of Rafsanjan; 2012. P.56

9. Gardner DG, Dyne LV, Pierce JL. The effects of pay level on organization-based Self-Esteem and performance: A field study. J Occup Organ Psychol 2004; 77(3):307-22.

10. De Lange AH, Taris TW, Kompier MAJ, Houtman ILD, Bongers PM. The relationship between characteristics and mental health: examining normal, reversed and reciprocal relationships in a 4wave study. Work Stress 2004; 18(2):149-66.

11. Davari A, Rezazadeh A. Modelling structural equations by PLS software. Tehran, Iran: Jehad Daneshgahi; 2016.

12. Stoeber J, Rambow A. Perfectionism in adolescent school student: relations with motivation, achievement, and well-being. Pers Individ Dif 2007; 42(7):1379-89.

13. Steel $P$. The nature of procrastination: a metaanalytic and theoretical review of quintessential self-regulatory failure. Psychol Bull 2007; 133(1):65-94.

14. Çapan BE. Relationship among perfectionism, academic procrastination and life satisfaction of university students. Procedia Soc Behav Sci 2010; 5:1665-71.

15. Abbaspour P, Farahani MN, Shahraray M. The Survey of Relationship between Perfectionism and Self-Esteem And Mental Health in Students of Tehran Medical Universities. Paper presented at: The Third Seminar of University Students' Mental Health; 2006 May 24-25; Tehran, Iran.

16. Pierce JL, Gardner DG. Self-Esteem within the work and organizational context: a review of the organization-based self-esteem Literature. J Manage 2004; 30(5):591-622 
17. Shek DT. Adolescent positive mental health and psychological symptoms: a longitudinal study in a Chinese context. Psychologia 1998; 41(4):217-25.

18. Ni C, Liu X, Hua Q, Lv A, Wang B, Yan Y. Relationship between coping, self-esteem, individual factors and mental health among Chinese nursing students: A matched case-control study. Nurse Educ Today 2010; 30(4):338-43.

19. Kunikata H, Shiraishi Y, Nakajima K, Tanioka T, Tomotake M. The relationship between psychological comfort space and self-esteem in people with mental disorders. J Med Invest 2011; 58(1-2):56-62.

20. Fialko L, Freeman D, Bebbington PE, Kuipers E, Garety PA, Dunn G, et al. Understanding suicidal ideation in psychosis: findings from the Psychological Prevention of Relapse in Psychosis (PRP) trial. Acta Psychiatr Scand 2006; 114(3):177-86. 